\title{
Bmi1, stem cells, and senescence regulation
}

\author{
In-Kyung Park, ${ }^{1}$ Sean J. Morrison, ${ }^{1,2,3}$ and Michael F. Clarke ${ }^{1,2}$ \\ ${ }^{1}$ Department of Internal Medicine, \\ ${ }^{2}$ Department of Cellular and Developmental Biology, and \\ ${ }^{3}$ Howard Hughes Medical Institute, University of Michigan, School of Medicine, Ann Arbor, Michigan, USA
}

Stem cells generate the differentiated cell types within many organs throughout the lifespan of an organism and are thus ultimately responsible for the longevity of multicellular organisms. Therefore, senescence of stem cells must be prevented. Bmi1 is required for the maintenance of adult stem cells in some tissues partly because it represses genes that induce cellular senescence and cell death.

J. Clin. Invest. 113:175-179 (2004). doi:10.1172/JCI200420800.

Many tissues are maintained throughout the lifespan of an organism by a small number of adult stem cells. These cells are unique in that they have both the ability to give rise to new stem cells via a process called selfrenewal and the ability to differentiate into the mature cells of a tissue. To maintain tissue homeostasis, stem cells have developed strict regulatory mechanisms to self-renew, differentiate, and prevent premature senescence and apoptosis (see review, ref. 1). The recent observation that Bmi1, a Polycomb group repressor, is essential for the self-renewal of adult murine hematopoietic stem cells (HSCs) and neuronal stem cells, in part via repression of genes involved in senescence, suggests that stem cells have evolved specific mechanisms to repress senescence and to prolong their capacity to proliferate. In this Perspective, we discuss the possible role of Bmi1 in the prevention of senescence in stem cells.

\section{What makes a cell a stem cell?}

HSCs are among the best-characterized stem cells. The existence of these cells was proven using clonal assays and retroviral marking $(2,3)$. Flow cytometry was then used to isolate HSCs based on cell-surface marker expression $(4,5)$. Subsequently, other types of somatic stem cells such as neuronal stem cells from the peripheral and central nervous systems have been identified $(6,7)$.

Stem cells possess three fundamental properties (1). First, they must self-renew, allowing the maintenance of the original stem cell population. Self-renewal is a

Address correspondence to: Michael F. Clarke, University of Michigan, School of Medicine, 1500 E. Medical Center Drive, Ann Arbor, Michigan 48109, USA. Phone: (734) 764-8195; Fax: (734) 647-9654; E-mail: mclarke@med.umich.edu.

Conflict of interest: The authors have declared that no conflict of interest exists.

Nonstandard abbreviations used: hematopoietic stem cell (HSC); senescence-associated heterochromatic foci (SAHF); retinoblastoma protein (pRB); acute myeloid leukemia (AML); mouse embryonic fibroblast (MEF); cyclin-dependent kinase (Cdk); mouse double minute 2 (MDM2); mammary epithelial cell (MEC). cell division in which one or both of the daughter cells are stem cells that retain the same developmental potential as the mother cell. In contrast, proliferation is a more general term that refers to all types of mitosis, whether they yield stem cells, restricted progenitors, or terminally differentiated cells. Second, stem cells must be able to differentiate into multiple types of mature cells in order to replace the mature cells that turn over in adult tissues. Third, the total number of stem cells is strictly regulated via both extrinsic and intrinsic mechanisms, resulting in the stability of a stable stem cell pool (8-11).

\section{Stem cells and senescence}

Senescence is a state in which a cell no longer has the ability to proliferate. Since stem cells maintain many tissues during the lifetime of an animal, it follows that stem cell senescence must be prevented to maintain an organ throughout life. Several studies suggest that cellular senescence is accompanied by changes in gene expression, which might be regulated by epigenetic mechanisms. In support of this hypothesis, histone deacetylase inhibitors, which decondense chromatin and activate the transcription of some genes, can induce a senescence-like state in human fibroblasts (12), suggesting that conversion of some heterochromatin to euchromatin may be a feature of replicative senescence $(13,14)$. Other studies suggest that chromatin condensation and subsequent downregulation of certain genes might regulate senescence. Senescence accompanies changes in nuclear morphology and formation of a distinct chromatin structure, called senescence-associated heterochromatic foci (SAHF) (15). SAHF do not contain active transcription sites, and they recruit heterochromatin proteins to the genes that are to be stably repressed during senescence. It was shown that SAHF contained the retinoblastoma protein ( $\mathrm{pRB}$ ) in the E2F-responsive promoters, such as cyclin $\mathrm{A}$ and proliferating cell nuclear antigen pro- 


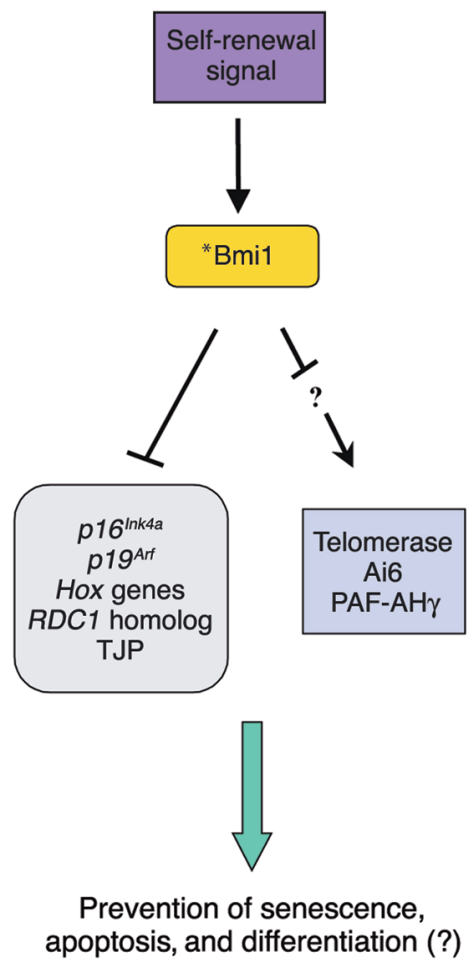

Figure 1

Postulated Bmi1 targets. Extrinsic signals for a stem cell to self-renew result in elevation of the Bmi1 level in stem cells. This allows repression of various genes including the $\ln k 4 a$ locus genes, $p 16^{\ln k 4 a}$ and p19 ${ }^{A r f}$, and possibly activation, via indirect mechanisms, of some genes including telomerase, apoptosis inhibitor-6 (Ai6), and plateletactivating factor acetylhydrolase (PAF-AH $\gamma$ ). These genes are likely play a role in stem cell fate decisions including self-renewal and differentiation. ${ }^{*}$ Sites of frequent mutations associated with cancer. TJP, tight junction protein. RDC1, chemokine orphan receptor 1 .

moters, and silenced the expression of E2F-responsive genes during senescence but not during quiescence (15). Formation of SAHF and silencing require an intact $\mathrm{PRB}$ pathway, since inhibition of $16^{\text {Ink4a }}$ prevents SAHF formation and leads to DNA replication. These results provide a molecular mechanism for the maintenance of the senescent state and demonstrate the importance of $\mathrm{pRB}$ as a tumor suppressor.

HSCs have an impressive regenerative potential, as demonstrated by transplantation experiments using limited numbers of cells. In mice, serial transplantation is possible for four to six passages, suggesting that individual HSCs are capable of extensive self-renewal but may not be immortal. Even though HSCs express telomerase $(16,17)$, it is not sufficient to completely prevent telomere erosion during aging (18). Overexpression of the catalytic subunit of the telomerase enzyme in hematopoietic cells prevents telomeres from shortening during serial transplantation of bone marrow. However, even HSCs overexpressing telomerase could be serially transplanted no more than four times, as is the case with wild-type HSCs; this suggests that a telomere-independent mechanism regulates replicative senescence of mouse HSCs during serial transplantation (19). On the other hand, telomerase-deficient HSCs can be serially transplanted only twice, accompanied by an increased rate of telomere shortening, indicating that telomerase is nonetheless needed to prevent premature loss of telomere function during serial transplantation $(20,21)$.

\section{Role of Bmi1 in stem cell self-renewal}

Since epigenetic events such as histone modification have been implicated in senescence, it follows that genes involved in chromatin remodeling and gene expression, such as members of the Polycomb and Trithorax families, might be directly involved in decisions that affect stem cell fate, including self-renewal, senescence, and possibly aging. Polycomb and Trithorax proteins form large multimeric structures, which can lead to repression or activation of gene expression, respectively, via a concerted process of chromatin modifications $(22,23)$.

Both HSCs and neuronal stem cells express high levels of Bmi1 (24-26), a member of the Polycomb group of transcription repressors that was initially identified as an oncogene cooperating with $c-m y c$ in a murine model of lymphoma $(27,28)$. Bmil has a RING finger at the amino-terminus and a central helix-turn-helix domain. The RING finger domain is required for the generation of lymphoma in E $\mu-B m i 1$ transgenic mice $(29,30)$. Postnatal mice lacking Bmi1 exhibit defects in hematopoiesis, skeletal patterning, neurological functions, and development of the cerebellum (31).

It has recently been shown that $B m i 1$ is necessary for efficient self-renewing cell divisions of adult HSCs as well as adult peripheral and central nervous system neural stem cells, but that it is less critical for the generation of differentiated progeny $(25,26)$. Transplantation of $\mathrm{Bmil}^{-/-}$fetal liver cells resulted in only transient hematopoietic cell reconstitution, suggesting that the transplanted mutant fetal liver HSCs failed to generate more HSCs but gave rise to multipotent progenitors that could sustain hematopoiesis for up to 4-8 weeks. Similarly, Bmi1 is needed for the maintenance of neural stem cells found in both the central and peripheral nervous systems. As with HSCs, the reduced self-renewal of Bmi1-deficient neural stem cells led to their postnatal depletion in vivo, but the proliferation and survival of committed progenitor cells were essentially normal (26). Given the broad ranges of phenotypic changes in Bmi1-deficient mice, including posterior transformation and neurological abnormalities (31), and its broad tissue distribution (32), it is likely that Bmi1 regulates the self-renewal of other types of somatic stem cells.

Bmi1 may also play a key role in some types of cancer (33-35). In approximately $11 \%$ of cases of mantle cell lymphoma, the malignant cells have a three- to sevenfold amplification of Bmi1 DNA and express high levels of the protein, implicating this gene in this invariably lethal form of lymphoma. In a mouse model of 
leukemia, Bmi1 was essential for the maintenance of leukemic cells (36). Enforced expression of Hoxa9/Meis-1 in both normal and Bmi1-deficient mouse fetal liver cells, followed by transplantation, initially resulted in infiltration of the bone marrow by cells that looked like acute myeloid leukemia (AML) blasts, and mice developed a bone marrow infiltrate that resembled AML. However, only Bmi1 wild-type AML could be serially transplanted. Taken together with the detection of high levels of Bmi1 in human AML stem cells (25), these results suggest that Bmil is also required for the selfrenewal of leukemic stem cells.

\section{Bmi1 and senescence}

In WI-38 human fetal lung fibroblasts, Bmi1 is downregulated when the cells undergo replicative senescence, but not when they are quiescent. Additionally, Bmi1 extends replicative lifespan but does not induce immortalization when overexpressed (37). In the absence of Bmi1, both the $p 16^{\text {Ink } 4 a}$ and the $p 19^{\text {Arf }}$ genes from the Ink4a locus are expressed (38). Lifespan extension by Bmi1 is mediated in part by suppression of the $p 16^{\text {Ink4a }}$ dependent senescence pathway and requires an intact pRB pathway, but not the p53 tumor-suppressor protein. The RING finger and helix-turn-helix domains of $B m i 1$ were required for lifespan extension and $p 16^{\text {Ink } 4 a}$ suppression. Furthermore, a RING finger deletion mutant acted as a dominant negative, inducing $p 16^{\text {Ink } 4 a}$ and premature senescence (37).

Normal mouse embryonic fibroblasts (MEFs) reach replicative senescence after seven passages in culture, whereas MEFs from $\mathrm{Bmil}^{-/-}$mice show a prematuresenescence phenotype at the third passage. This was correlated with increased expression of $p 16^{\text {Ink } 4 a}$. Reexpression of Bmil in $\mathrm{Bmi1}^{-/-}$MEFs prevented premature senescence (28). Overexpression of Bmi1 gave a proliferative advantage and extended MEF lifespan. Furthermore, unlike human fibroblasts, Bmi1 could immortalize MEFs.

\section{Downstream targets of Bmi1}

Gene-profiling studies suggest that Bmi1 modulates HSC self-renewal through the regulation of genes important for stem cell fate decisions, as well as survival genes, antiproliferative genes, and stem cellassociated genes (Figure 1) (25). The previously mentioned Bmil target, the Ink4a locus (28), encodes $p 16^{\text {Ink4a }}$ and $p 19^{\text {Arf }}$ using different promoters (38). Enforced expression of $p 16^{\text {Ink4a }}$ and $p 19^{A r f}$ in HSCs led to senescence and apoptosis, respectively (25). In neural stem cells, $p 16^{\text {Ink4a }}$ deficiency partially restored the ability of Bmi1-deficient stem cells to self-renew (26). Figure 2 illustrates regulation of the cell cycle and senescence by $16^{\text {Ink4a }}$ and p19Arf. During the cell cycle, $\mathrm{pRB}$ is hyperphosphorylated by the cyclin D/ cyclin-dependent kinases 4 and 6 (cyclin D/Cdk4/6) complex (39). The hyperphosphorylated $\mathrm{pRB}$ is unable to bind and inhibit E2F transcription factor, allowing transcription of E2F target genes that are important for the G1/S transition, such as DNA polymerase II, cyclin E, p19, myb, and dihydrofolate reductase (40). This allows cell cycle progression. In the absence of Bmi1, p16 $6^{\text {Ink4a }}$ is upregulated and prevents binding of Cdk4/6 to cyclin D, inhibiting the kinase activity. This results in hypophosphorylated $\mathrm{pRB}$, which then binds E2F and inhibits E2F-mediated transcription, leading to cell cycle arrest and senescence (39). p19Arf sequesters mouse double minute 2 (MDM2) and inhibits p53 degradation, resulting in p53-mediated cell cycle arrest and apoptosis $(41,42)$. Point mutations and deletion of $p 16^{\text {Ink4a }}$ and $p 19^{\text {Arf }}$ are frequently found

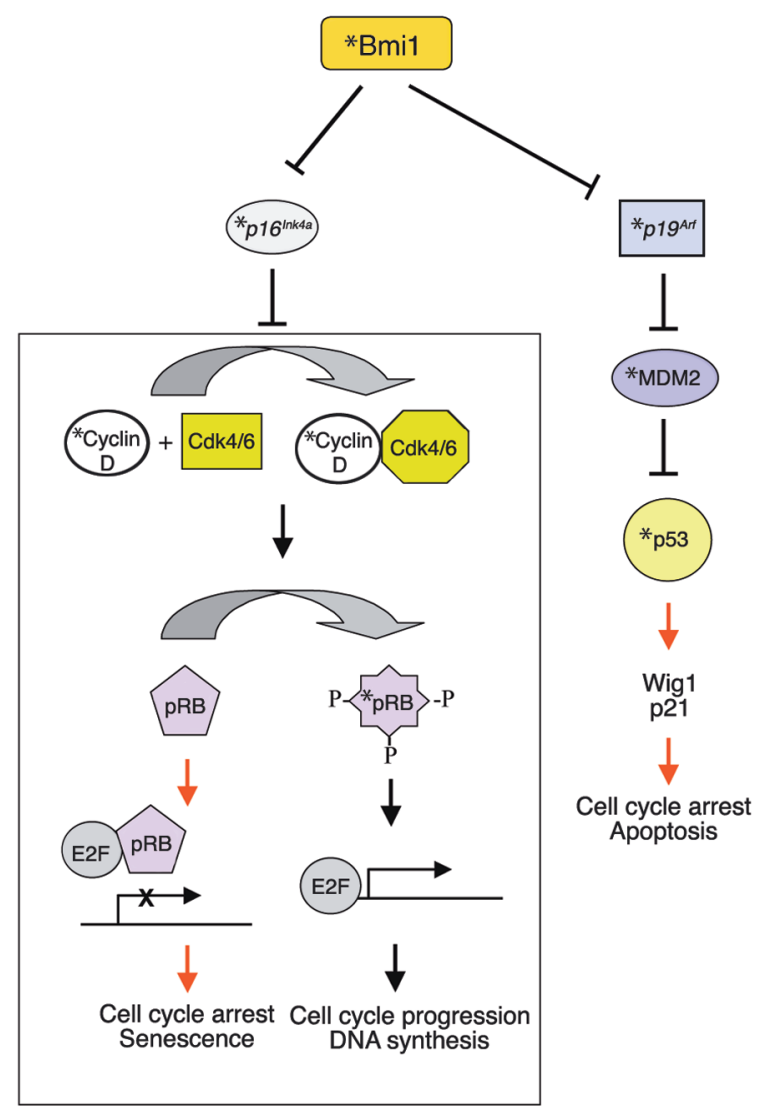

\section{Figure 2}

Regulation of cell cycle, apoptosis, and senescence by Bmi1. In normal stem cells, $p 16^{\operatorname{lnk} 4 a}$ and $p 19^{\text {Arf }}$ genes are repressed in a Bmi1dependent manner. In the absence of $p 16^{\ln k 4 a}$, the cyclin D/Cdk4/6 complex can phosphorylate pRB, allowing the E2F-dependent transcription that leads to cell cycle progression and DNA synthesis. In addition, MDM2-mediated p53 degradation causes low p53 levels in the absence of p19Arf, thus preventing cell cycle arrest and apoptosis. The absence of Bmi1 relieves the repression of the Ink $4 a$ locus, resulting in the expression of $p 16^{\ln k 4 a}$ and $p 19^{A r f}$. $p 16^{\ln k 4 a}$ inhibits binding of cyclin D to Cdk4/6, resulting in inhibition of the kinase activity. This leads to a hypophosphorylated pRB, which then can bind E2F and inhibit E2F-dependent transcription, resulting in cell cycle arrest and senescence. p19Arf inhibits MDM2, which mediates ubiquitin-dependent degradation of $\mathrm{p} 53$, thus leading to accumulation of $\mathrm{p} 53$ protein in the cell. This leads to induction of various p53 target genes involved in cell cycle arrest and apoptosis. Proteins affected by high and low levels of Bmi1 are shown by black and red arrows, respectively. *Sites of frequent mutations associated with cancer. 
in many types of human cancers, which implicates them as key regulators of immortalization and/or senescence checkpoints.

Mice lacking Bmi1 showed induction of both $p 16^{\text {Inkta }}$ and $p 19^{A r f}$ in various hematopoietic and neuronal tissues (25). Overexpression of $p 16^{\text {Ink } 4 a}$ and $p 19^{A r f}$ in adult HSCs induced cell cycle arrest and apoptosis via the $\mathrm{pRB}$ and the p53-dependent pathway, respectively. Double deletion of the Bmi1 and $p 16^{\text {Ink4a }} / p^{1} 9^{\text {Arf }}$ genes partially rescued the phenotypes observed in Bmi1-deficient mice (28), suggesting that $p 16^{\text {Ink4a }}, p 19^{A r f}$, and p53 are downstream effectors of $B m i 1$ that are involved in the control of the proliferation and survival of HSCs during self-renewing cell divisions (Figure 2). Therefore, Bmi1 maintains the HSC pool in part by repressing genes involved in cellular senescence. Increased expression of the p53 target gene Wig1 in $\mathrm{Bmil}^{-/}$bone marrow suggests that the $p 19^{\text {Arf }}$ pathway may have been activated in $\mathrm{Bmil}^{-/-}$hematopoietic cells. Wig1 is a double-stranded RNA-binding protein and inhibits tumor growth in vitro, suggesting that it may function in stress-induced p53 responses (43). The observation that $\mathrm{p} 53$-deficient mice have increased numbers of stem cells is consistent with the notion that p53 might be a downstream effector of Bmi1 (44). In addition, some of the Hox 9 family of genes are also affected in Bmi1-deficient hematopoietic tissues and neurospheres $(25,26)$. Determination of the relative contribution of each of these pathways to the regulation of HSC selfrenewal will require careful analysis of the HSCs from double- or triple-knockout mice.

There is evidence that Bmi1 might regulate telomerase expression in human mammary epithelial cells (MECs) and might play a role in the development of human breast cancer. Bmi1 is overexpressed in several breast cancer cell lines and postselection human MECs immortalized with human papilloma virus $E 6$ oncogene, which abrogates the $\mathrm{p} 53 / \mathrm{p} 21^{\text {waf }}$ pathway (45), suggesting that Bmil might be involved in immortalization. Postselection MECs can be obtained by regular feeding of a heterogeneous population of MECs from primary mammary tissue. During this process, the $p 16^{\text {Inkta }}$ gene is progressively silenced and not expressed in postselection MECs $(46,47)$. Overexpression of Bmi1 in postselection MECs bypasses senescence, extending replicative lifespan and immortalizing MECs. This is associated with human telomerase reverse transcriptase (hTERT) expression, which leads to induction of telomerase activity. Although hTERT is a direct target of c-Myc-induced transcription in MECs (48, 49), Bmi1 appeared to act independently of c-Myc. Since Bmi1 is a transcription repressor, induction of telomerase is probably mediated by an indirect mechanism. Deletion analysis of the Bmi1 protein suggested that the RING finger, as well as the conserved helix-turn-helix domain, was required for its ability to induce telomerase and immortalization. These data suggest that Bmi1 directly or indirectly regulates telomerase expression in MECs and might play a role in the development of human breast cancer. However, Bmi1 induction of telomerase is cell type specific; Bmi1 fails to induce telomerase in fibroblasts (45). This is consistent with the observation that Bmil overexpression did not immortalize human fibroblasts (37). It is not known whether Bmi1 is involved in telomere function in normal breast stem cells.

\section{Future directions}

Bmi1 maintains the stem cell pool by preventing premature senescence, either through repression of genes involved in senescence or perhaps through induction of telomerase to prevent telomere shortening. It is very likely that Bmi1 is important for maintenance of multiple types of somatic stem cells, since it is widely expressed and Bmi1-deficient mice have developmental defects in other organs. Bmi1 is also important for maintenance of leukemic stem cells and perhaps other tumorigenic stem cells; therefore, Bmil could be used as a molecular target to induce senescence in cancer stem cells (50).

Since Bmi1 maintains the HSC pool size and regulates key genes implicated in senescence and aging, it is of interest to determine whether expression of Bmi1 and its target genes changes during stem cell transplantation and/or aging. Whether stem cells undergo senescence during aging is controversial (51-53). In C57BL mice, in which most HSC studies have been performed, HSC numbers increase with age without losing overall function (54-56). However, HSC senescence might occur during aging in certain other strains of mice $(57,58)$. The number of times that HSCs can reconstitute the bone marrow of lethally irradiated mice is limited in serial-transplantation experiments. This observation might be either a result of an intrinsic stem cell aging program that occurs only when stem cell proliferation far exceeds that seen during normal aging, or a result of damage to the stem cells that is secondary to the stress of the transplant. In either model, it is possible that the loss of stem cell activity is mediated by Bmi1 or its downstream targets.

1. Reya, T., Morrison, S., Clarke, M., and Weissman, I. 2001. Stem cells, cancer, and cancer stem cells. Nature. 414:105-111.

2. Till, J., and McCulloch, E. 1961. A direct measurement of the radiation sensitivity of normal mouse bone marrow cells. Radiat. Res. 14:1419-1430.

3. Williams, D., Lemischka, I., Nathan, D., and Mulligan, R. 1984. Introduction of new genetic material into pluripotent haematopoietic stem cells of the mouse. Nature. 310:476-480.

4. Spangrude, G.J., Heimfeld, S., and Weissman, I.L. 1988. Purification and characteristics of mouse hematopoietic stem cells. Science. 241:58-62.

5. Morrison, S.J., and Weissman, I.L. 1994. The long-term repopulating subset of hematopoietic stem cells is deterministic and isolatable by phenotype. Immunity. 1:661-673.

6. Stemple, D., and Anderson, D. 1992. Isolation of a stem cell for neurons and glia from the mammalian neural crest. Cell. 71:973-985.

7. Davis, A., and Temple, S. 1994. A self-renewing multipotential stem cell in embryonic rat cerebral cortex. Nature. 372:263-266.

8. Domen, J., Cheshier, S.H., and Weissman, I.L. 2000. The role of apoptosis in the regulation of hematopoietic stem cells: overexpression of BCL-2 increases both their number and repopulation potential.J. Exp. Med. 191:253-264.

9. Lemischka, I.R., and Moore, K.A. 2003. Stem cells: interactive niches. Nature. 425:778-779.

10. Calvi, L.M., et al. 2003. Osteoblastic cells regulate the haematopoietic stem cell niche. Nature. 425:841-846. 
11. Zhang, J., et al. 2003. Identification of the haematopoietic stem cell niche and control of the niche size. Nature. 425:836-841.

12. Ogryzko, V., Hirai, T., Russanova, V., Barbie, D., and Howard, B. 1996 Human fibroblast commitment to a senescence-like state in response to histone deacetylase inhibitors is cell cycle dependent. Mol. Cell. Biol. 16:5210-5218.

13. Howard, B. 1996. Replicative senescence: considerations relating to the stability of heterochromatin domains. Exp. Gerontol. 31:281-293.

14. Villeponteau, B. 1997. The heterochromatin loss model of aging. Exp. Gerontol. 32:383-394.

15. Narita, M., et al. 2003. Rb-mediated heterochromatin formation and silencing of E2F target genes during cellular senescence. Cell. 113:703-716.

16. Pathak, S. 2002. Organ- and tissue-specific stem cells and carcinogenesis. Anticancer Res. 22:1353-1356.

17. Morrison, S., Prowse, K., Ho, P., and Weissman, I. 1996. Telomerase activity in hematopoietic cells is associated with self-renewal potential. Immunity. 5:207-216.

18. Yui, J., Chiu, C.-P., and Lansdorp, P.M. 1998. Telomerase activity in candidate stem cells from fetal liver and adult bone marrow. Blood. 91:3255-3262.

19. Allsopp, R.C., and Weissman, I.L. 2002. Replicative senescence of hematopoietic stem cells during serial transplantation: does telomere shortening play a role? Oncogene. 21:3270-3273.

20. Samper, E., et al. 2002. Long-term repopulating ability of telomerasedeficient murine hematopoietic stem cells. Blood. 99:2767-2775.

21. Allsopp, R.C., Morin, G.B., DePinho, R., Harley, C.B., and Weissman, I.L. 2003. Telomerase is required to slow telomere shortening and extend replicative lifespan of HSCs during serial transplantation. Blood. 102:517-520.

22. Simon, J., and Tamkun, J. 2002. Programming off and on states in chromatin: mechanisms of Polycomb and trithorax group complexes. Curr. Opin. Genet. Dev. 12:210-218.

23. Orlando, V. 2003. Polycomb, epigenomes, and control of cell identity. Cell. 112:599-606.

24. Lessard, J., Baban, S., and Sauvageau, G. 1999. Stage-specific expression of Polycomb group genes in human bone marrow cells. Blood. 91:1216-1224.

25. Park, I.-K., et al. 2003. Bmi1 is required for maintenance of adult selfrenewing haematopoietic stem cells. Nature. 423:302-305.

26. Molofsky, A.V., et al. 2003. Bmi1 dependence distinguishes neural stem cell self-renewal from progenitor proliferation. Nature. 425:962-967.

27. Haupt, Y., Bath, M., Harris, A., and Adams, J. 1993. Bmi1 transgene induces lymphomas and collaborates with myc in tumorigenesis. Oncogene. 8:316-314.

28. Jacob, J., Kieboom, K., Marino, S., Depinho, R., and van Lohuizen, M 1999. The oncogene and Polycomb-group gene Bmi1 regulates cell proliferation and senescence through the ink4a locus. Nature. 397:164-168.

29. van Lohuizen, M., et al. 1991. Identification of cooperating oncogenes in E mu-myc transgenic mice by provirus tagging. Cell. 65:737-752.

30. Alkema, M., Jacobs, H., van Lohuizen, M., and Berns, A. 1997. Pertubation of B and $\mathrm{T}$ cell development and predisposition to lymphomagenesis in Emu Bmi1 transgenic mice require the Bmi1 RING finger. Oncogene. 15:899-910.

31. van der Lugt, N.M., et al. 1994. Posterior transformation, neurological abnormalities, and severe hematopoietic defects in mice with a targeted deletion of the Bmi1 proto-oncogene. Genes Dev. 8:757-769.

32. Haupt, Y., Alexander, W., Barri, G., Klinken, S., and Adams, J. 1991. Novel zinc finger gene implicated as myc collaborator by retrovirally accelerated lymphomagenesis in E mu-myc transgenic mice. Cell. 65:753-763.

33. Bea, S., et al. 2001. BMI1 gene amplification and overexpression in hematological malignancies occur mainly in mantle cell lymphomas. Cancer Res. 61:2409-2412.

34. van Kemenade, F.J., et al. 2001. Coexpression of BMI1 and EZH2 poly- comb-group proteins is associated with cycling cells and degree of malig nancy in B-cell non-Hodgkin lymphoma. Blood. 97:3896-3901.

35. Vonlanthen, S., et al. 2001. The Bmi1 oncoprotein is differentially expressed in non-small cell lung cancer and correlates with INK4A-ARF locus expression. Br.J. Cancer. 84:1372-1376.

36. Lessard, J., and Sauvageau, G. 2003. Bmi1 determines the proliferative capacity of normal and leukaemic stem cells. Nature. 423:255-260.

37. Itahana, K., et al. 2003. Control of the replicative life span of human fibroblasts by 16 and the polycomb protein Bmi1. Mol. Cell. Biol. 23:389-401.

38. Quelle, D.E., Zindy, F., Ashmun, R.A., and Sherr, C.J. 1995. Alternative reading frames of the INK4a tumor suppressor gene encode two unrelated proteins capable of inducing cell cycle arrest. Cell. 84:993-1000.

39. Sharpless, N., and DePinho, R. 1999. The INK4A/ARF locus and its two gene products. Curr. Opin. Genet. Dev. 9:22-30.

40. Vernell, R., Helin, K., and Muller, H. 2003. Identification of target genes of the p16INK4A-pRB-E2F pathway. J. Biol. Chem. 278:46124-46137.

41. Weber, J.D., Taylor, L.J., Roussel, M.F., Sherr, C.J., and Bar-Sagi, D. 1999. Nucleolar Arf sequesters Mdm2 and activates p53. Nat. Cell Biol. 1:20-26.

42. Honda, R., and Yasuda, H. 1999. Association of p19ARF with Mdm2 inhibits ubiquitin ligase activity of $\mathrm{Mdm} 2$ for tumor suppressor $\mathrm{p} 53$. EMBO J. 18:22-27.

43. Mendez-Vidal, C., Wilhelm, M.T., Hellborg, F., Qian, W., and Wiman, K.G. 2002. The p53-induced mouse zinc finger protein wig- 1 binds double-stranded RNA with high affinity. Nucleic Acids Res. 30:1991-1996.

44. TeKippe, M., Harrison, D.E., and Chen, J. 2003. Expansion of hematopoietic stem cell phenotype and activity in Trp53-null mice. Exp. Hematol. 31:521-527.

45. Dimri, G.P., et al. 2002. The Bmi1 oncogene induces telomerase activity and immortalizes human mammary epithelial cells. Cancer Res. 62:4736-4745.

46. Brenner, A., Stampfer, M., and Aldaz, C. 1998. Increased p16 expression with first senescence arrest in human mammary epithelial cells and extended growth capacity with p16 inactivation. Oncogene. 17:199-205.

47. Wong, D., Foster, S., Galloway, D., and Reid, B. 1999. Progressive regionspecific de novo methylation of the $\mathrm{P} 16 \mathrm{CPG}$ island in primary human mammary epithelial cell strains during escape from $\mathrm{M}(0)$ growth arrest. Mol. Biol. Cell. 19:5642-5651.

48. Wu, K., et al. 1999. Direct activation of TERT transcription by c-MYC Nat. Genet. 21:220-224.

49. Greenberg, R., et al. 1999. Telomerase reverse transcriptase gene is a direct target of c-Myc but is not functionally equivalent in cellular transformation. Oncogene. 18:1219-1226.

50. Schmitt, C. 2003. Senescence, apoptosis and therapy: cutting the lifelines of cancer. Nat. Rev. Cancer. 3:286-295.

51. Van Zant, G., and Liang, Y. 2003. The role of stem cells in aging. Exp. Hematol. 31:659-672.

52. Liang, Y., and Van Zant, G. 2003. Genetic control of stem-cell properties and stem cells in aging. Curr. Opin. Hematol. 10:195-202.

53. Geiger, H., True, J.M., de Haan, G., and Van Zant, G. 2001. Age- and stage-specific regulation patterns in the hematopoietic stem cell hierarchy. Blood. 98:2966-2972.

54. Morrison, S., Prowse, K., Ho, P., and Weissman, I. 1996. The aging of hematopoietic stem cells. Nat. Med. 2:1011-1016.

55. Sudo, K., Ema, H., Morita, Y., and Nakauchi, H. 2000. Age-associated characteristics of murine hematopoietic stem cells. J. Exp. Med. 192:1273-1280.

56. Kim, M., Moon, H.-B., and Spangrude, G.J. 2003. Major age-related changes of mouse hematopoietic stem/progenitor cells. Ann N. Y. Acad. Sci. 996:195-208

57. Van Zant, G., Holland, B., Eldridge, P., and Chen, J. 1990. Genotyperestricted growth and aging patterns in hematopoietic stem cell populations of allophenic mice. J. Exp. Med. 171:1547-1565.

58. Chen, J., Astle, C.M., and Harrison, D.E. 2000. Genetic regulation of primitive hematopoietic stem cell senescence. Exp. Hematol. 28:442-450. 